\title{
Tubal assessment with Hysterosalpingography following routine Intravenous Buscopan: A need to avoid unnecessary radiation and discomfort
}

\author{
Akintomide A. $\mathrm{O}^{1}$, Eduwem D.U ${ }^{2}$, Ikpeme A.A ${ }^{3}$, Bassey D.E. ${ }^{4}$ \\ ${ }^{1,4}$ Department of Radiology, faculty of medicine and dentistry, College of medical sciences, \\ University Of Calabar, Cross-River, Nigeria
}

\begin{abstract}
Background: The main indication for hysterosalpingography (HSG) is infertility, primarily to assess the fallopian tubes. Its major drawback is tubal spasm, which is preventable by administering intravenous (IV) Buscopan prior to the procedure. This is not routinely done by all practitioners, rather they administer it after the tubes are not demonstrated, which leads to repeats. HSG involves a relatively high radiation dose to the pelvic organs compared to none contrast examinations, so multiple attempts of the procedure and unnecessary radiation exposure should be discouraged.

Objectives:Administer intravenous Buscopan prior to the procedure and assess the fallopian tubes, adverse drug reaction and the number of attempts of the procedure.

Subjects and Methods: A prospective study of 130 women, aged between 19 and 42 years over two years, at 2 radiodiagnostic units. We administered IV Buscopan before the procedure.

Results: We observed HSG abnormalities in 94(72.3\%) women and 32(24.6\%) showed multiple site involvement. Sixty-one (46.9\%) women had abnormalities in the tubes(commonest sites), mainly due to occlusion.

Conclusion: We did our procedures once, thus avoiding additional radiation exposure and discomfort due to repeated examinations in suspected tubal spasm when Buscopan is administered later to relieve it. No serious adverse reaction to Buscopan.
\end{abstract}

Keywords:Buscopan, Fallopian tubes, Hysterosalpingography, Infertility, Spasm.

\section{Introduction}

The main indication for Hysterosalpingogram (HSG) is infertility ${ }^{[1]}$, primarily to evaluate the fallopian tubes ${ }^{[2]}$. Tubal occlusion was the commonest abnormality seen during HSG by Khatiet al ${ }^{[3]}$ and Al subhiet $a l^{[4]}$.The actual incidence of true positive tubal occlusion during HSG will not be accurate, if precautions like administration intravenous buscopanis not taken to prevent the non-demonstration of the fallopian tubes due to spasm. In a study by Adrian et al, tubal spasm accounted for a high false positive rate of $39 \%$ for proximal tubal occlusion ${ }^{[5]}$.

HSG was first performed in 1910 by Rindfleisch using Bismuth solution ${ }^{[6]}$. Over the years, the procedure has undergone modifications in terms of technique, instrumentation and types of contrast medium to improve its diagnostic capability, safety and comfort, while reducing the pain associated with it. Conventional HSG remains the imaging modality of choice to assess the fallopian tubes despite the advances made in various modalities such as a three-dimensional dynamic magnetic resonance hysterosalpingography (3D dMR-HSG) and contrast enhanced hystero-salpingo-sonography ${ }^{[7]}$. These two have higher resolution and are non-ionizing.

The status of fallopian tubes has become more important in recent years because of the availability of advanced fertility treatment options for female infertility. For occluded fallopian tubes, the interventional radiologist can carry out a selective salpingography and tubal recanalization, which allow numerous pregnancies that would otherwise have required in vitro fertilization (IVF) or tubal microsurgery.

Suggestions on ways to reduce the false positive rate of tubal occlusion include the administration of 10 or 20 milligram (mg) of intravenous (IV)buscopan if the tubes are not demonstrable and repetition of theexamination ${ }^{[2]}$. Some researchers gave a time frame to repeat the examination, while some did not. Irrespective of the time given, the patient goes through discomforting process of cannulation and/or contrast injection twice and spends a longer time in the awkward lithotomy position ${ }^{[3,5,8]}$. Two other workers in the reviewed literature however advised that, buscopan should be given prior to the procedure ${ }^{[9,10]}$. The aim of this 
study is to establish the safety of Buscopan and the possibility of a single attempt of HSG, which reduces the procedure time and radiation dose. The HSG findings are also analysed.

\section{Subjects and Methods}

This is a prospective descriptive study involving all 130 women, aged $19-42$ years, undergoing routine workup for primary and secondary infertility over a two-year period (September 2013 and September 2015) at the radiology department of Nigerian Navy hospital and a specialist radio-diagnostic centre in south south, Nigeria.The procedures were scheduled based on the "Ten day rule" between the $6^{\text {th }}$ and $10^{\text {th }}$ day of their menstrual cycle after cessation of menses.

\subsection{Inclusion criteria}

1. Women referred to the two centres for HSG as part of their infertility work-up.

2. Women already investigated and/or treated for PID.

\subsection{Exclusion criteria}

1. Previous tubal surgery including salpingectomy for ectopic pregnancy.

2. Women with signs and symptoms of pelvic inflammatory disease.

3. Active vaginal bleeding.

\subsection{Technique}

As a general guideline for HSG procedure, we obtained an informed consent from the patients. Contraindications to Buscopan such as glaucoma, myasthenia gravis, megacolon and known hypersensitivity to hyoscinebutylbromide or its other components were ruled-out. After emptying the bladder, the patient undress and put on a gown with an open back. The preliminary representative pelvic radiograph was obtained in supine position before the administration of $20 \mathrm{mg}$ of IV Buscopan. The patient is then put in the lithotomy position for the procedure. Using aseptic technique, the speculum lubricated with K Y jelly, was inserted into the vagina to locate the cervix, which is cleaned with antiseptic. The cervical lip was held with two pairs of forceps(vulcellum or tenaculum) at 9 and 3 O'clock. The contrast medium used is $15-30 \mathrm{mls}$ of $76 \%$ Urograffin (water soluble, ionic) in 3:2 dilution with normal saline depending on the capacity of the endometrial cavity. The Urograffinwas injected slowly through the leech Wilkinson cannula of the appropriate size after air bubbles were expelled. The first representative contrast radiograph was obtained after $5 \mathrm{mls}$ of contrast injection to outline the endometrial cavity, the second after injection of another 10 to $15 \mathrm{mls}$ to outline a well distended endometrial cavity, the fallopian tubes and early peritoneal spill of contrast. A delayed radiograph demonstrates the pattern of peritoneal spill. Oral analgesics and buscopan were prescribed for a day or two after the procedure and the patients were observed for adverse reaction to Buscopan for 30 minutes before leaving the department. Results of the HSG findings were analysed with simple statistical methods and presented in percentages, tables and figures.

\section{Results}

Themean age of the 130 women recruited into the study was 32.7 years and most of them were within the $26-30$ years age group. Fourteen $(10.8 \%)$ presented with primary infertility and $116(89.2 \%)$ with secondary infertility. Fig. 1 shows the distribution of normal and abnormal HSG. Thirty-two (24.6\%) women had multiple site pathologies.

Table 1 shows the pathologies at the different sites on HSG with the tubes being the commonest; $61(46.9 \%)$ women. These tubal abnormalities were either unilateral or bilateral but in all, the right and the left were involved 49(37.7\%) and 45(34.6\%) times respectively.

Table 2 shows the distribution of the tubal findings in our study. Single tubal abnormality either occlusion or dilatation (hydrosalpinx) occurred in $27(20.8 \%)$ women, while $34(26.1 \%)$ had bilateral tubal abnormalities; occlusion, hydrosalpinx or a combination of both. There were 53(40.8\%) women with tubal occlusion and 19(14.6\%) with hydrosalpinx(unilaterally and bilaterally).

Fig. 2 shows the distribution of tubal occlusion while Fig. 3 shows that of hydrosalpinx. The right tube was occluded (Fig. 4) in $42(32.3 \%)$ women and dilated (Fig. 5) in 12 (9.2\%) women, while the left was occluded (Fig. 5) in $40(30.8 \%)$ and dilated (Fig. 6) in 13 (10\%) women. Fig 7 shows the image of bilateral tubal occlusion on HSG. 


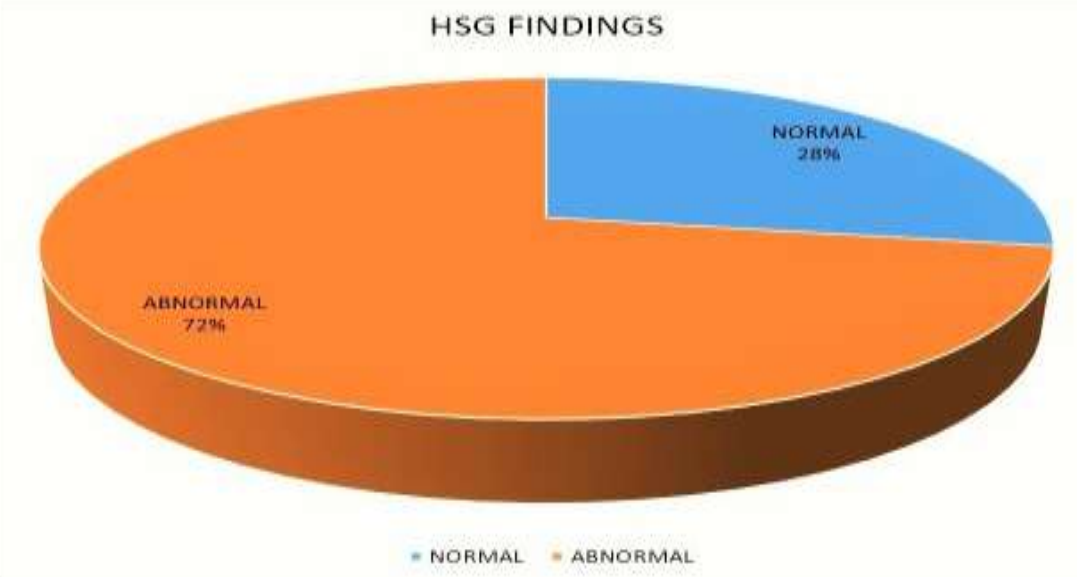

Figure 1. Distribution of normal and abnormal hysterosalpingogram.

HSG: Hysterosalpingography.

Table 1.Distribution of the sites and pathologies of the abnormal hysterosalpingographic findings ( $\mathrm{n}=94)$.

\begin{tabular}{|c|c|c|c|}
\hline $\mathrm{S} / \mathrm{n}$ & Sites/Pathologies & Frequency (n) & Percentage $(\%)$ \\
\hline 1 & $\begin{array}{l}\text { Cervical canal } \\
\text {. Stenosis } \\
\text {. Cervicitis }\end{array}$ & $\begin{array}{l}2 \\
2\end{array}$ & $\begin{array}{l}2.1 \\
2.1\end{array}$ \\
\hline 2 & $\begin{array}{l}\text { Uterus } \\
\text { Intramural fibroid } \\
\text {. Submucous fibroid } \\
\text {. Synechiae }\end{array}$ & $\begin{array}{l}8 \\
28 \\
18\end{array}$ & $\begin{array}{l}8.5 \\
29.8 \\
19.2\end{array}$ \\
\hline 3 & $\begin{array}{l}\text { Fallopian tubes: Occlusion and Hydrosalpinx } \\
\text {. Right } \\
\text {. Left } \\
\text {. Bilateral }\end{array}$ & $\begin{array}{l}16 \\
11 \\
34\end{array}$ & $\begin{array}{l}17.0 \\
11.7 \\
36.2\end{array}$ \\
\hline 4 & Pelvic cavity: Adhesions & 10 & 10.6 \\
\hline
\end{tabular}

Note: 32 women showed multiple sites abnormalities.

Table 2.Distribution of hysterosalpingographic tubal findings.

\begin{tabular}{|l|l|l|}
\hline Findings & Frequency (n) & Percentage (\%) \\
\hline Normal tubes & 69 & 53 \\
\hline Right tubal Occlusion & 10 & 7.7 \\
\hline Right tubal Occlusion and bilateral hydrosalpinges & 1 & 0.8 \\
\hline Right tubal Occlusion and left hydrosalpinx & 2 & 1.5 \\
\hline Left tubal Occlusion & 9 & 6.9 \\
\hline Left tubal Occlusion and bilateral hydrosalpinges & 1 & 0.8 \\
\hline Left tubal Occlusion and left hydrosalpinx & 1 & 0.8 \\
\hline Bilateral tubal Occlusion & 23 & 17.7 \\
\hline Bilateral tubal Occlusion and bilateral hydrosalpinges & 4 & 3.1 \\
\hline Bilateral tubal Occlusion and left hydrosalpinx & 2 & 1.5 \\
\hline Right hydrosalpinx & 6 & 4.6 \\
\hline Left hydrosalpinx & 2 & 1.5 \\
\hline
\end{tabular}






Figure 2. Distribution of tubal occlusion. TBLK - Tubal blockage (occlusion).

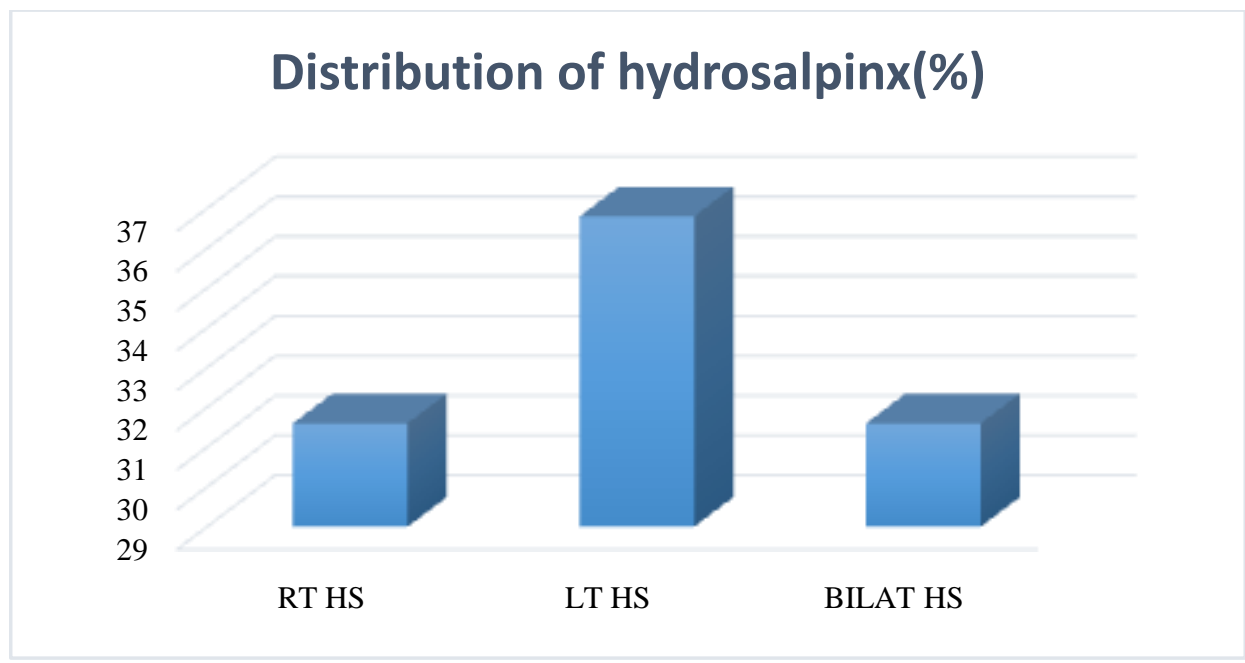

Figure 3. Distribution of hydrosalpinx HS - Hydrosalpinx.

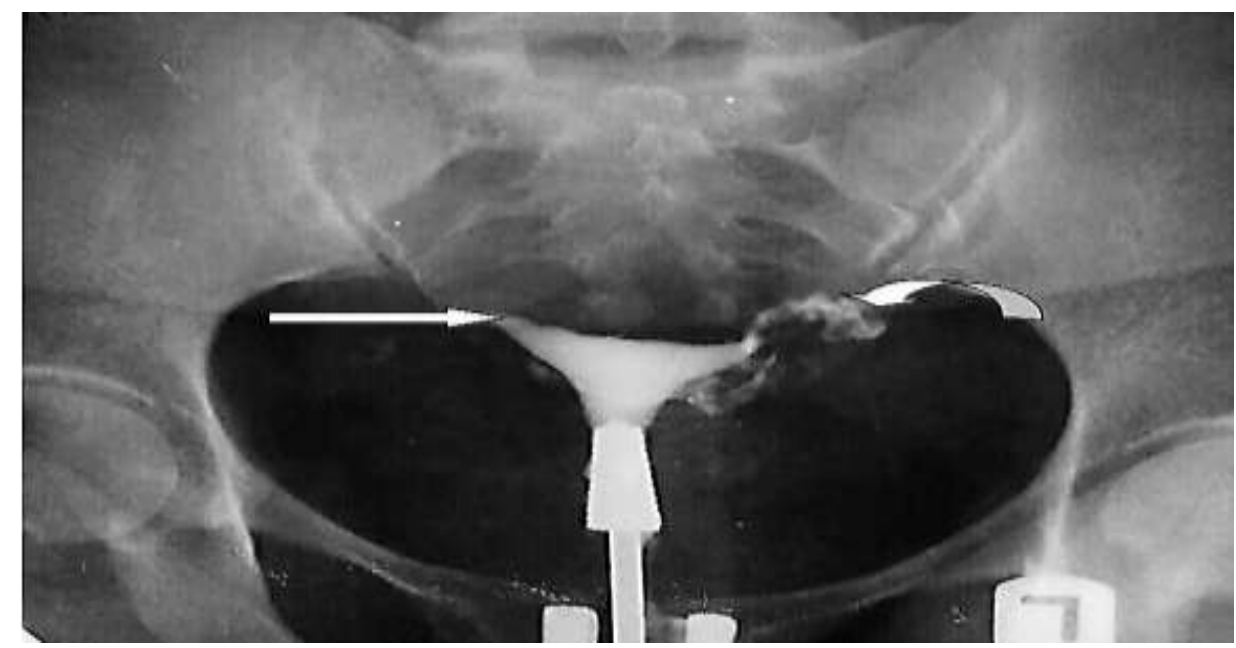

Figure 4. Hysterosalpingogram showing a right tubal occlusion (straight arrow) and a normal left tube (curved arrow). 


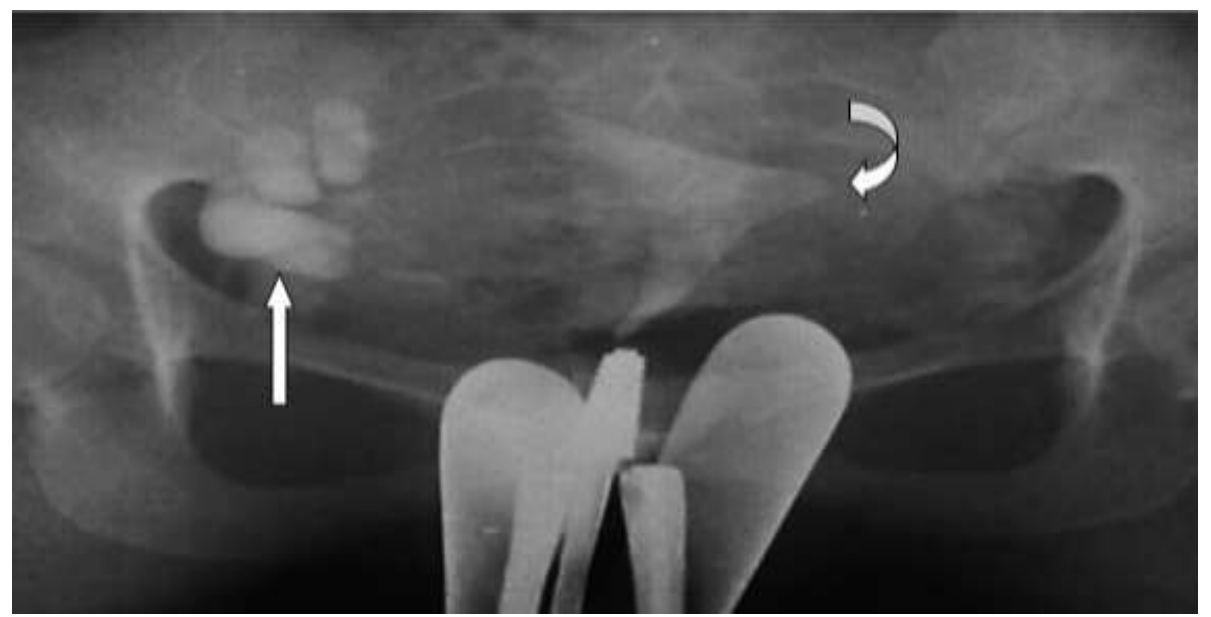

Figure 5.Hysterosalpingogram showing moderate right hydrosalpinx (straight arrow) and a left tubal occlusion (curved arrow).

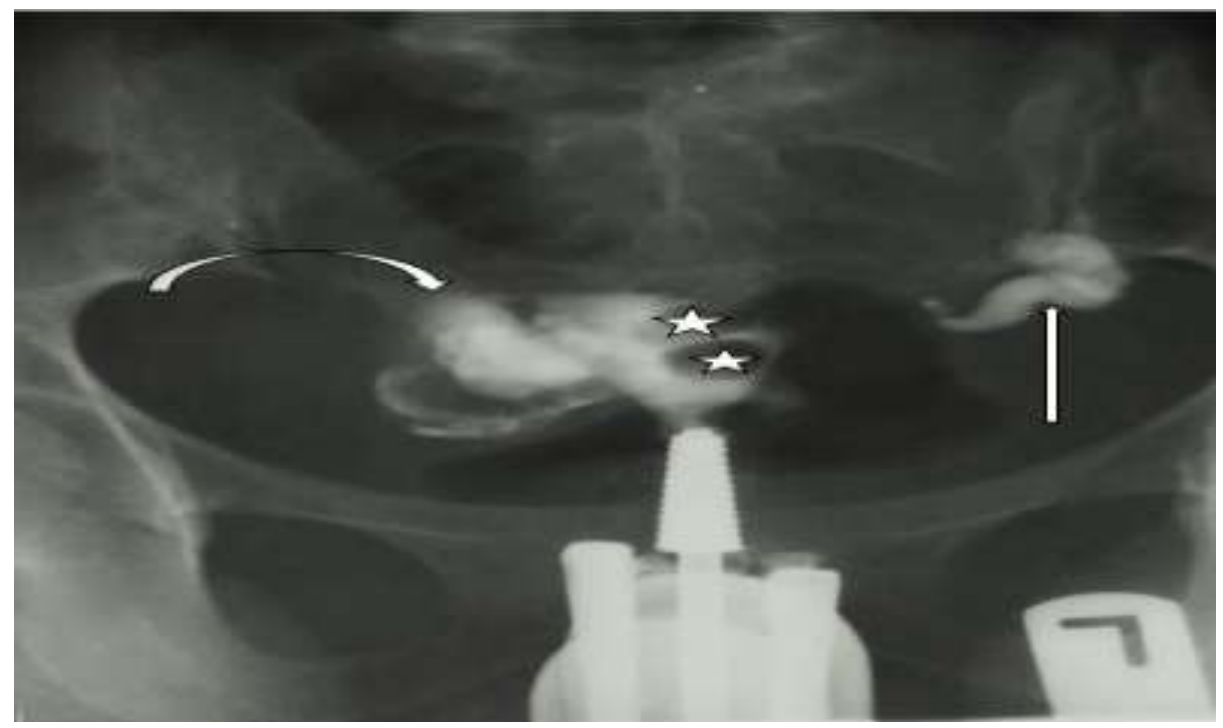

Figure 6.Hysterosalpingogram showing two endometrial nodular filling defects (stars), mild dilatation of the left fallopian tube distally (straight arrow) and a right peritoneal spill of contrast (curved arrow).

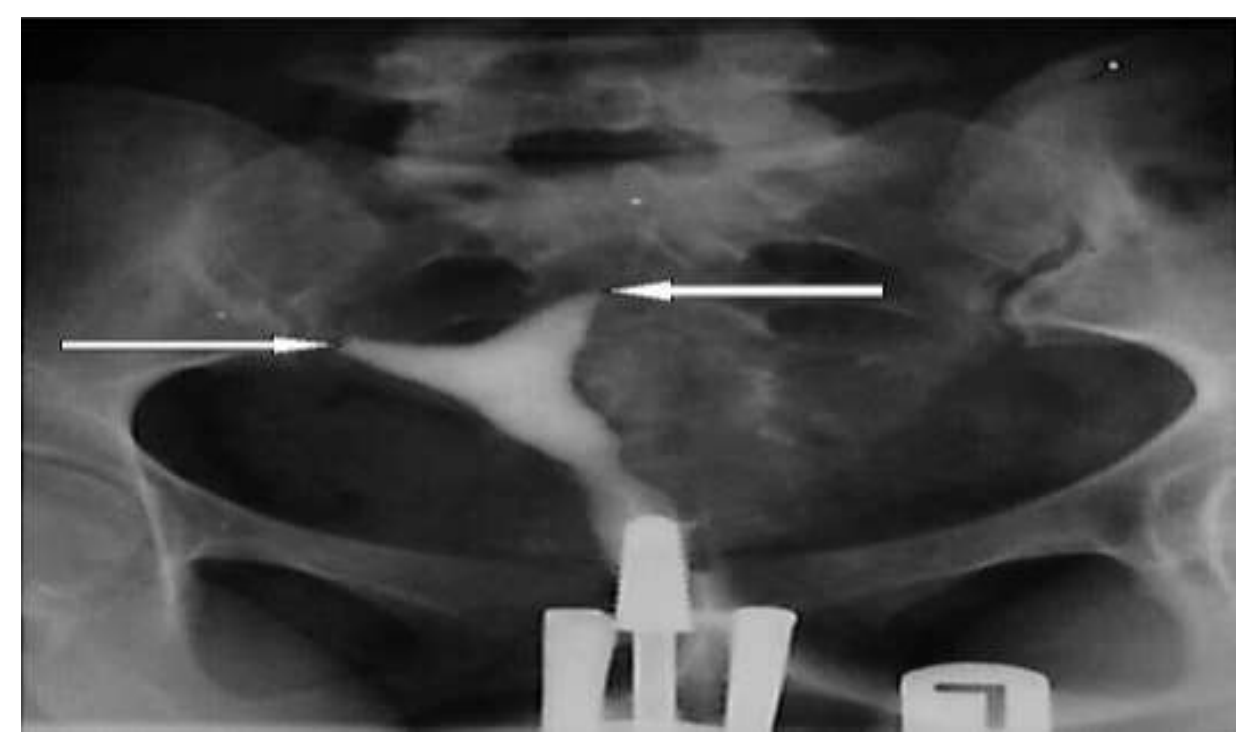

Figure 7. Hysterosalpingogram showing bilateral tubal occlusion (straight arrows) 


\section{Discussion}

Administration of the IV Buscopan prior to HSG allows a single attempt of the procedure and an early decision on the state of tube, thus avoiding unnecessary additional radiation exposure and discomfort if the procedure was repeated when the tubes are not demonstrated. Occlusion of the fallopian tube(s) are common pathologies seen during HSG. However, the true incidence of tubal occlusion will not be accurate with HSG if tubal spasm is not ruled-out. Buscopan Is known to be effective in relieving tubal spasm as demonstrated by Adrian et alwho observed tubal patency after it administration in nine cases that previously showed occlusion ${ }^{[5]}$. Based on this, we gave intravenous buscopan $(20 \mathrm{mg})$ just before the onset of the procedure to reduce the incidence of false positive tubal occlusion due to tubal spasm.

The other precautions taken based on the advice of fellow researchers are prior counselling to allay fears and gentle handling ${ }^{[9]}$. We achieved this, usingthin clamps on the cervical lip that will not snap when torqued on and the clamps were applied at two opposite sides ( 9 and 3 ' 0 clock) to properly stabilize the external cervical os for easy sounding of the uterus, cervical cannulation and injection of contrast. We also injected the contrast medium slowly ${ }^{[1]}$ to reduce or eliminate the irritation of the endometrium.

If we did not give Buscopan initially, we would have extended the procedure time to give it later, inject more contrast medium or re-cannulate the cervix for a repeat in the $46(35.5 \%)$ women in our study who had tubal occlusion without hydrosalpinx. This is in addition to the unknown number in whom the Buscopan had already prevented spasm. These would have led to more ionizing radiation, discomfort and pain.

Our study showed normal HSG findings in 36(27.7\%) patients which is similar to the $29.1 \%$ and $29.4 \%$ observed by Okafor et al ${ }^{[11]}$ and Bukar et al ${ }^{[12]}$ respectively. This is higher than the $21.8 \%$ observed by Imo and Adeoye ${ }^{[13]}$ and the $20 \%$ by Ibekwe et al ${ }^{[14]}$,but lower than the $70 \%$ observed by VR Shrivastava ${ }^{[15]}$ and $37.9 \%$ by Bacevac and Ganoxic ${ }^{[16]}$. We observed that the fallopian tubes are the commonest sites of pathology being involved in $46.9 \%$ of the women, which is comparable to the findings by two studies; $43.5 \%$ and $44.1 \%{ }^{[11,17]}$ but lower than two others; $72.1 \%$ and $54.6 \%{ }^{[12,13]}$. Santosh et al however had a significantly lower rate of tubal abnormalities of $20 \%{ }^{[18]}$. We observed that the right tube $(12.3 \%)$ is more frequently involved in pathologies than the left $(8.5 \%)$.

The incidence of tubal occlusion in our study was high (40.8\%) compared to other studies; $28.2 \%$, $28.6 \%$ and $32 \%{ }^{[10,18,19]}$ probably due to the reported high prevalence of vaginal infection in this environment, which commonly results in chronic pelvic inflammatory disease (PID) if not treated promptly ${ }^{[20,21]}$.

The incidence of bilateral tubal pathology $(25.4 \%)$ in our study is higher than unilateral $(15.4 \%)$. This observation is similar to that of two studies ${ }^{[13,19]}$. However, four other studies showed a higher incidence of unilateral tubal pathology $y^{[15,17,18,22]}$. There is a higher incidence of tubal blockage than hydrosalpinx, which is similar to the findings of others ${ }^{[5,12,23]}$.Igashi et al observed an equal incidence between the two ${ }^{[17]}$.

\section{Conclusion}

Our study showed a high burden of the tubal factor, especially occlusion in female infertility. This incidence is likely due to the high rate of vaginal infection in this environment. All our procedures where done once, thus eliminating undesirable additional radiation exposure and discomfort if the examinations were repeated. We prevented an extended or repeated procedure in at least the $46(35.4 \%)$ women who had tubal occlusion without hydrosalpinx. There was no serious adverse reaction to buscopan in any of our patients. We therefore recommend routine intravenous Buscopan for patients prior to HSG, when it is not contra-indicated.

\section{REFERENCES}

[1]. ACR Practice parameter for the performance of Hysterosalpingography Resolution 39

[2]. Simpson W, Beitia LG, Mester F. Hysterosalpingography: A re-emerging study. Radiograhphics 2006; $26: 419$ - 31.

[3]. Khati Nadia J, Remer Jeffrey, Brindle Kathleen. Hysterosalpingography findings in women with infertility. Contemporary Diagnostic Radiology: 2012. 35; 11:1-6.

[4]. Al subhi T, Al Jashnmi RN, Al Khaduri M, Gowri V, Prevalence of tubal obstruction In the hysterosalpingogram of women with primary and secondary infertility. J Reprod infertile. 2013 Oct; 14(4): $214-6$.

[5]. Adrian C. Schankath, Nikola Fasching, Cornelia Urech-Ruh, Michael K. Hohl, Rahel A. Kubik-Huch. Hysterosalpingography in the workup of female infertility: indications, technique and diagnostic findings. Insights imaging (2012) 3: 475 - 483.

[6]. Rindfleisch W. Darstellung des cavum uteri. KlinWochenscher. 1910; 47: 780.

[7]. Unterweger M, De Geyter C, Frohlich JM. Three-dimensional dynamic MR-hysterosalpingography; a new, low invasive, radiationfree and less painful radiological approach to female infertility. Hum Reprod. 2002; 17(12): 3138 - 41.

[8]. H MohdNor, KJ Jayapragasam, BJJ Abdullahi. Diagnostic image quality of hysterosalpingography: Ionic versus non-ionic water soluble iodinated contrast media. Biomed imaging interv. J. 2009 Jul-sept; 5(3): e29.

[9]. Das S, Nardo LG, Seif MW. Proximal tubal disease: the place for cannulation. Reprod Biomed online 2007; 15(4): 383 - 8 . 
[10]. M. Siva Sridhar, D Sowjanya, D. Sai Raghavendra. Sonosalpingography (SSG) - As a safe alternative to hysterosalpingogram (HSG) in evaluating female infertility. JEMDS: 2014: Vol 3 (32): Pg 8786-8790.

[11]. Okafor C.O, Okafor C.I, Okpala O.C, Umeh E. The pattern of hysterosalpingographic findings in women being investigated for infertility in Nnewi Nigeria. Niger J ClinPract. 2010 Sep; 13(3): 264 - 7.

[12]. Bukar M, Mustapha Z, Takai UI, Tahir A. Hysterosalpingograhic findings in infertile women: a seven-year review. Niger J ClinPract. 2011 Apr - Jun; 14 (2): 168 - 70. doi: 10.4103/1119 - 3077. 84008.

[13]. Imo A. O, Sunday - Adeoye I. Radiological assessment of the Uterus and fallopian tubes in infertile women at Abakaliki, Nigeria. Niger J ClinPract. 2008 Sep; 11 (3): 211 - 5

[14]. Ibekwe PC, Udensi AM, Imo AO. Hysterosalpingoraic findings in patients with infertility in south-eastern Nigeria. Niger J Med 2010; 19: $165-167$.

[15]. VR Shrivastava, B Rijal, A Shrestha, HK Shrestha and AS Tuladhar. Detection of tubal abnormalities by HSG in Nepalese subfertile women. Nepal Med Coll, 2009; 11(1): $42-45$.

[16]. Bacevac J, Ganoxic R. Diagnostic value of hysterosalpingography in examination of fallopian tubes in infertile women. SrpArhCelokLek. 2001 Sep - Oct; 129 (9 - 10): 243 -6.

[17]. Joseph Bako Igashi, Dlama Zira Joseph, Gloria Joseph, SaiduKadas, GeofreyLuntsi, FlaviousNkubli, Samuel Laushugno, ObettaChinedu T. Hysterosalpingography: A re-emerging study in health care transformation. IOSR Journal of dental and medicine sciences (IOSR- JDMS) JDMS e-ISSN: 2279 - 0853, p- ISSN: 2276 - 0861. Volume 13, issue 12 Ver, VII (Dec. 2014), PP 37 - 40.

[18]. Santosh Rai P V, Pallavi Rao, Harikiran Reddy. Hysterosalpingography (HSG) - 'The last man standing' among the erstwhile plethora of conventional radiological investigations. 'How we do it' and review of literature. International journal of medical and applied sciences (E- ISSN: 2320 - 3137) Volume 3, issue 4, 2014.

[19]. Waheed S, Mazhar R, Khan N.H, Rafi M. The comparison of hysterosalpingography and laparoscopy in predicting fertility. Annals Vol 13. No 3 jul - sept 2007; $202-205$.

[20]. J.A Lennox, S.D. Abbey, D. Udiba, C.I Mboto, I.S Ikpoh, F.C Akuguenyi. Prevalence of vaginitis and vaginosis among university of Calabar female students. Journal of public health and epidemiology. Vol. 5(4) pp. 167 - 172, April 2013.

[21]. P.D Ekwere, E.I Archibong, E.E Bassey, J.E Ekabua, E.I Ekanem and P. Feyi-waboso. Infertility among Nigerian couples as seen in Calabar. Port Harcourt Medical Journal 2007; 2: 35 - 40.

[22]. Eng CW, Tang PH, Ong CL. Hysterosalpingography. Current applications. Singapore Med J 2007; 48: 368 - 73.

[23]. Bello T.O. Pattern of tubal pathology in infertile women on hysterosalpingography in Ilorin, Nigeria. Annals of African medicine Vol. 3, No 2; 2004: 77 - 79 . 